\title{
INTERFERENCE ALIGNMENT TESTBEDS
}

\author{
Cenk M. Yetis, Academia Sinica, Taiwan \\ Jacobo Fanjul, University of Cantabria, Spain \\ José A. García-Naya, University of A Coruña, Spain \\ Nima N. Moghadam, KTH Royal Institute of Technology, Sweden \\ Hamed Farhadi, Harvard University, USA
}

\begin{abstract}
Interference alignment (IA) has triggered high impact research in wireless communications since it was proposed nearly ten years ago. However, the vast majority of research are centered on the theory of IA and are hardly feasible in view of the existing state-of-the-art wireless technologies. Although several research groups assessed the feasibility of IA via testbed measurements in realistic environments, the experimental evaluation of IA is still in its infancy since most of the experiments were limited to simpler scenarios and configurations. This article summarizes the practical limitations of experimentally evaluating IA, provides an overview of the available IA testbed implementations, including the costs, and highlights the imperatives for the succeeding IA testbed implementations. Finally, the article explores future research directions on the applications of IA in the next generation wireless systems.
\end{abstract}

\section{INTRODUCTION}

The recently developed interference alignment (IA) concept revealed that the throughput of a wireless network can be significantly improved compared to the one exhibited by conventional transmission schemes such as time-division multiple access (TDMA) or frequency-division multiple access (FDMA). Unfortunately, it is extremely difficult to take into account all practical limitations in the analytical investigation of IA, yielding theoretical results to be frequently based on assumptions which are hardly realizable in real-world scenarios. Examples of such practical aspects are the impacts of imperfect channel state information (CSI), energy loss due to spatial collinearity between desired signal and interference subspaces, detection and synchronization errors, and imperfect hardware. Consequently, the experimental evaluation of IA techniques is crucial to better understand the impacts of the aforementioned practical limitations on the performance of existing IA techniques as well as to propose new research topics around the IA concept to overcome such limitations. This is precisely the main goal of this article, which consists in proposing future research directions aiming for adopting IA to an attractive solution to be considered by the industry for the next generations of wireless communication systems.

The original IA concept assumes perfect CSI knowledge in all terminals to design the corresponding beamformers and filters. In practice, however, the users can acquire only a noisy version of the CSI, yielding a significant performance degradation in terms of the achievable sum degrees of freedom (DoF), as shown in [1] for a pilot-assisted channel estimation technique in a $K$-user interference network with single-antenna users. The CSI acquisition problem is mitigated in time-division duplexing (TDD) 
systems by exploiting channel reciprocity, although calibrated RF equipment is required [2]. For frequency-division duplexing (FDD) systems, IA experiments with perfect [3], [4] and realistic analog wireless [5] feedback channels have been reported in the literature. Furthermore, in most of the theoretical IA works, the block-fading channel model assumption plays a pivot role due to its mathematical tractability [1]. In practice, guaranteeing a constant channel during a block transmission is not possible, leading also to the problem of outdated CSI at the transmitters.

The implementation of a perfect IA scheme requires a large number of transmitters (e.g., base stations in the downlink of cellular networks) and network resources (i.e., time, frequency, number of antennas, and power). Particularly, the number of signal space dimensions grows exponentially with the number of users. Hence, in a $K$-user interference network, $K$ transmitters are required to serve $K$ users. Therefore, further research is needed on multiple-input multiple-output (MIMO) interference relay broadcast channels to serve more users per transmitter via relays with confined resources.

The finite signal-to-noise ratio (SNR) at the receiver is another practical limitation. However, experimental results show that, despite the imperfections in both CSI acquisition and testbed hardware, IA outperforms conventional communication schemes such as TDMA and greedy interference avoidance in the mid-to-high SNR regime [4]. However, an optimal trade-off between network resources dedicated to CSI acquisition and feedback with respect to those devoted to data transmissions must be determined to maximize the throughput [1], [6], [7]. Error vector magnitude (EVM) experimental results for a pilot-assisted maximum signal-to-interference plus noise ratio (max-SINR) scheme corroborate the existence of an optimal resource allocation scheme [7], and an optimal number of training symbols [4].

Time and frequency synchronization between network nodes is of utmost importance when experimentally evaluating IA techniques and it can be implemented in a centralized [8] or a distributed manner [2], [5]. Theoretical IA works usually assume that beamformers operate after frame detection and synchronization. In practical systems, however, frame detection and synchronization are applied immediately after the analog-to-digital conversion, hence being affected by interference and yielding a strong performance degradation of such tasks, thus impacting dramatically on the final system performance. For the specific case of spatial IA in multicarrier systems, IA decoding can be implemented in the time domain or following a more conventional per-subcarrier approach in the frequency domain [3], [4]. Given that time-domain IA decoding suppresses most of the multiuser interference at the very beginning of the receiver signal processing chain, the effective SINR is improved, whereas synchronization tasks perform similar to the interference-free case because they operate after filtering the interference out [4].

Hardware imperfections are ignored in many of the IA algorithm designs. However, nonlinear distortions, phase noise, IQ imbalance and frequency offset degrade the IA performance [3], [9]. For instance, the measurement results of IA in the 3-user $2 \times 2$ MIMO interference channel show that hardware imperfections can reduce the maximum achievable SINR up to $10 \mathrm{~dB}$ compared to the theoretical predictions [9]. To compensate for the leaked interference under non-ideal conditions, power control is suggested as a complementary interference management technique [10].

This article addresses the main practical limitations that have been found when experimentally evaluating IA, and describes solutions to mitigate their impacts on the IA performance. The rest of the article is structured as follows. First, a panorama of the testbeds that have been employed to validate different IA techniques is provided. An estimation of the cost of these testbeds and statistics regarding the publications associated to them are provided. Finally, the article explores future directions to be taken by both theoretical and experimental IA investigations for enabling IA in the next generation of wireless communications. 


\section{HIGHLIGHTS OF TESTBED IMPLEMENTATIONS}

In this section we provide statistical information on IA experiments and publications, as well as financial costs of these IA implementations. Various options that span from low- to high-end solutions are also summarized.

\section{Publications on IA Experiments}

Some statistics regarding the number of IA publications are shown in Fig. 1 and 2. As the IA concept evolves, testbeds incorporate a mixture of dedicated hardware components and commercial off-the-shelf modules (we refer to them as dedicated platforms). When the concept matures, the amount of dedicated hardware components in the testbed platforms overrun. As shown in Fig. 1, commercial off-the-shelf products are still the leading choices for IA implementations within the research community. On the other hand, as seen in Fig. 2, the number of IA testbed publications fluctuates from year to year, and each year a fairly good number of experimental studies are published.

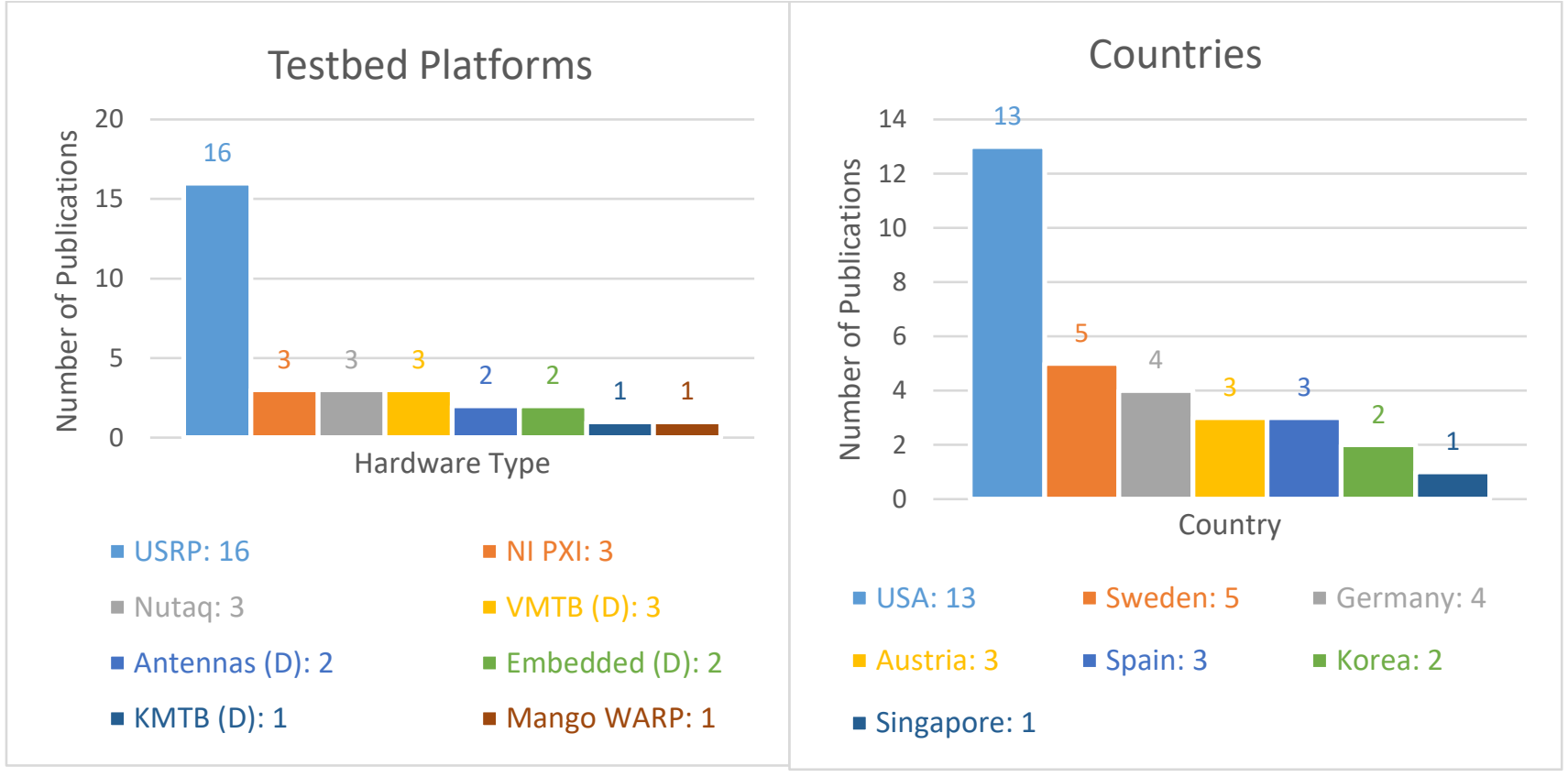

Figure 1. From left to the right, the number of IA testbed publications (only officially published papers are considered) per hardware type and per country are given, respectively. D: stands for partially dedicated testbed platforms, where there are specifically designed hardware components along with offthe-shelf products, KMTB: stands for Kista MIMO testbed in Sweden, VMTB: stands for Vienna MIMO testbed in Austria, antennas: stands for testbed implementations with antennas that have different radiation patterns, and finally, embedded: stands for embedded implementations. 


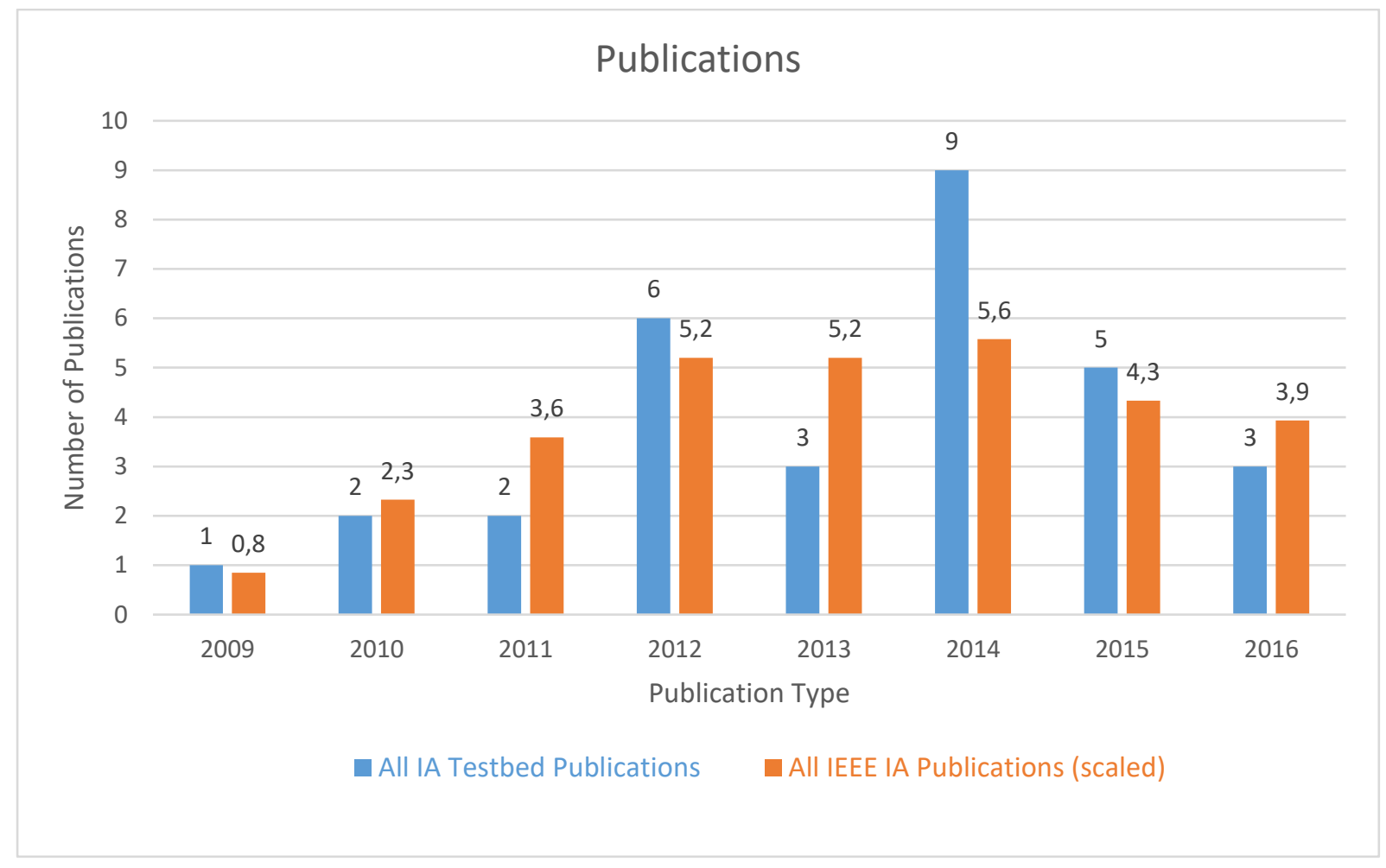

Figure 2. All IA testbed publications (including non-IEEE publications) and the scaled number of all IEEE IA publications (including non-testbed publications) per year are given with blue and brown colors, respectively. The scaling factor 47 is obtained from the ratios of the averages of theoretical and implementation papers per year.

\section{Analysis of the Costs of Equipment in IA Experiments}

In Table 1, the equipment that are used in each of the reported IA testbeds and its estimated costs are listed. As can be seen in the list, a very affordable IA setup is demonstrated in [8] where transmit antenna selection is applied and two antennas out of three are selected. The next affordable setup is reported in [5]. With a similar total cost, high-performance USRPs support $2 \times 2$ MIMO configurations, as shown in Table 1. The first IA real-time implementation is introduced in [11], where blind interference alignment (BIA) is implemented for a 2 -user $2 \times 1$ broadcast channel. In this setup, there are two antennas at the transmitter, whereas at the receiver, one antenna out of two is selected. Except [8] and [11], all configurations in Table 1 are outlined for the 3 -user $2 \times 2$ interference channel with a single stream per user, $(2 \times 2,1)^{3}$, including the equipment used in the recent implementation of massive MIMO testbed by the University of Bristol and Lund University collaboration. With a comparable total cost and testbed setup, a similar equipment list is used in the centralized PXI configuration. Finally, in the distributed PXI configuration, a separate chassis is used for each transmitter. 
Table 1. The quantity needed per equipment and the total estimated cost are listed for exemplary IA testbeds and for some possible IA testbed configurations. For simplicity, only the main equipment is listed, software and other equipment costs, including cables, antennas, and PCs are not listed in the table. The rounded prices are in thousands of US dollars and obtained from the National Instruments US website.

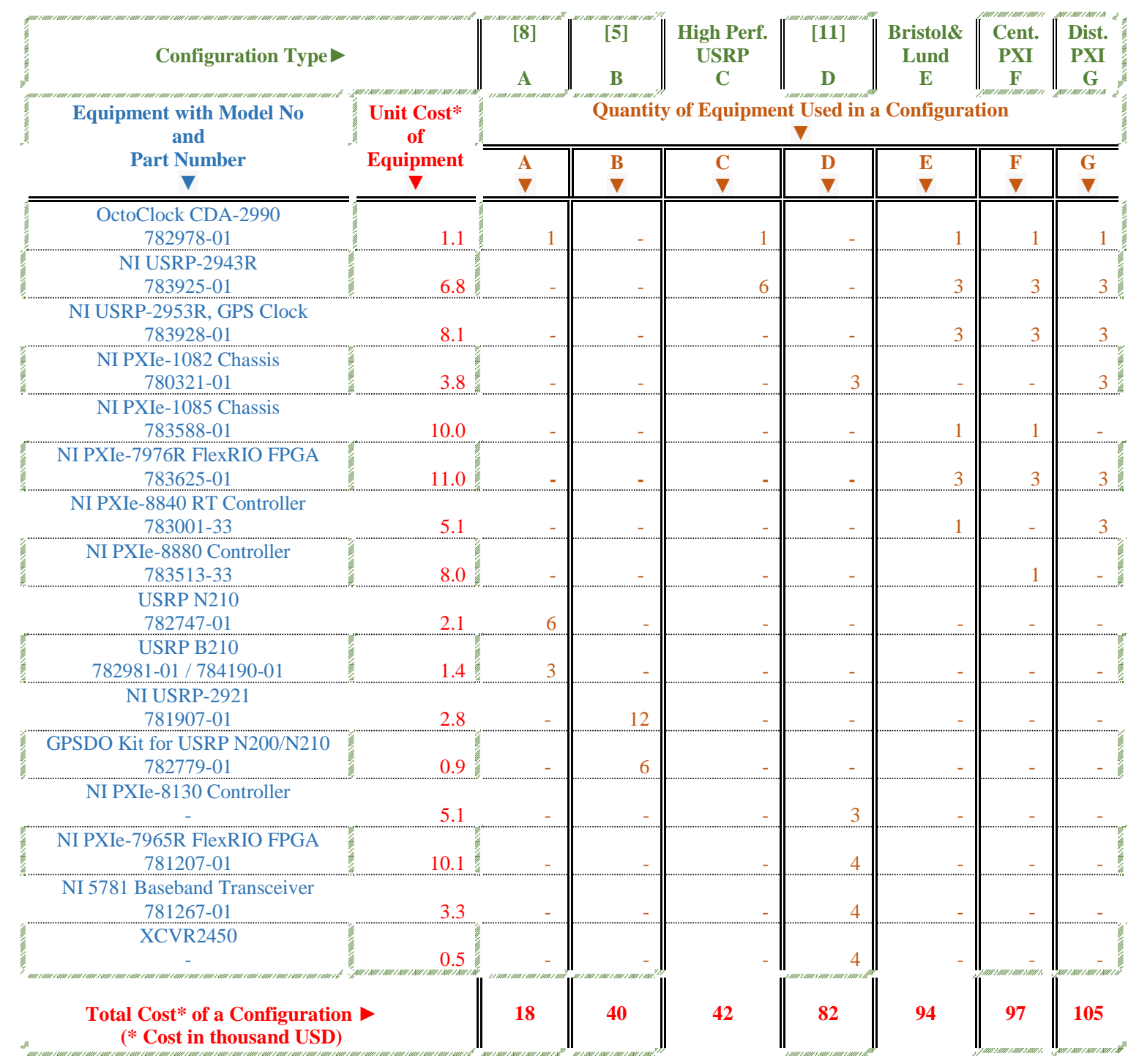

In Fig. 3, the estimated costs of selected configurations from Table 1, namely 1) configuration C: high performance USRPs, 2) configuration F: centralized PXI, and 3) configuration G: distributed PXI are plotted versus the network size. In the massive MIMO demonstration by the universities in Bristol and Lund, 16 users are served by a 128 -antenna transmitter. Accordingly, in order to implement IA in the $(9 \times 8,1)^{16}$ scenario, by using the same equipment listed in Table 1 for the configurations $\mathrm{F}$ and $\mathrm{G}$, a total cost of nearly two million US dollars is estimated. As shown in Fig. 3, to scale the network DoF by a factor of four, i.e., from $3:(2 \times 2,1)^{3}$ to $12:(8 \times 7,3)^{4}$, the cost is expected to increase nearly four times. Whereas by scaling the DoF approximately by a factor of five, i.e., from 3 to $16:(9 \times 8,1)^{16}$, the cost is expected to scale by a factor of 20 . Hence, for large network sizes where IA is significantly competitive, large capital investments are required. 


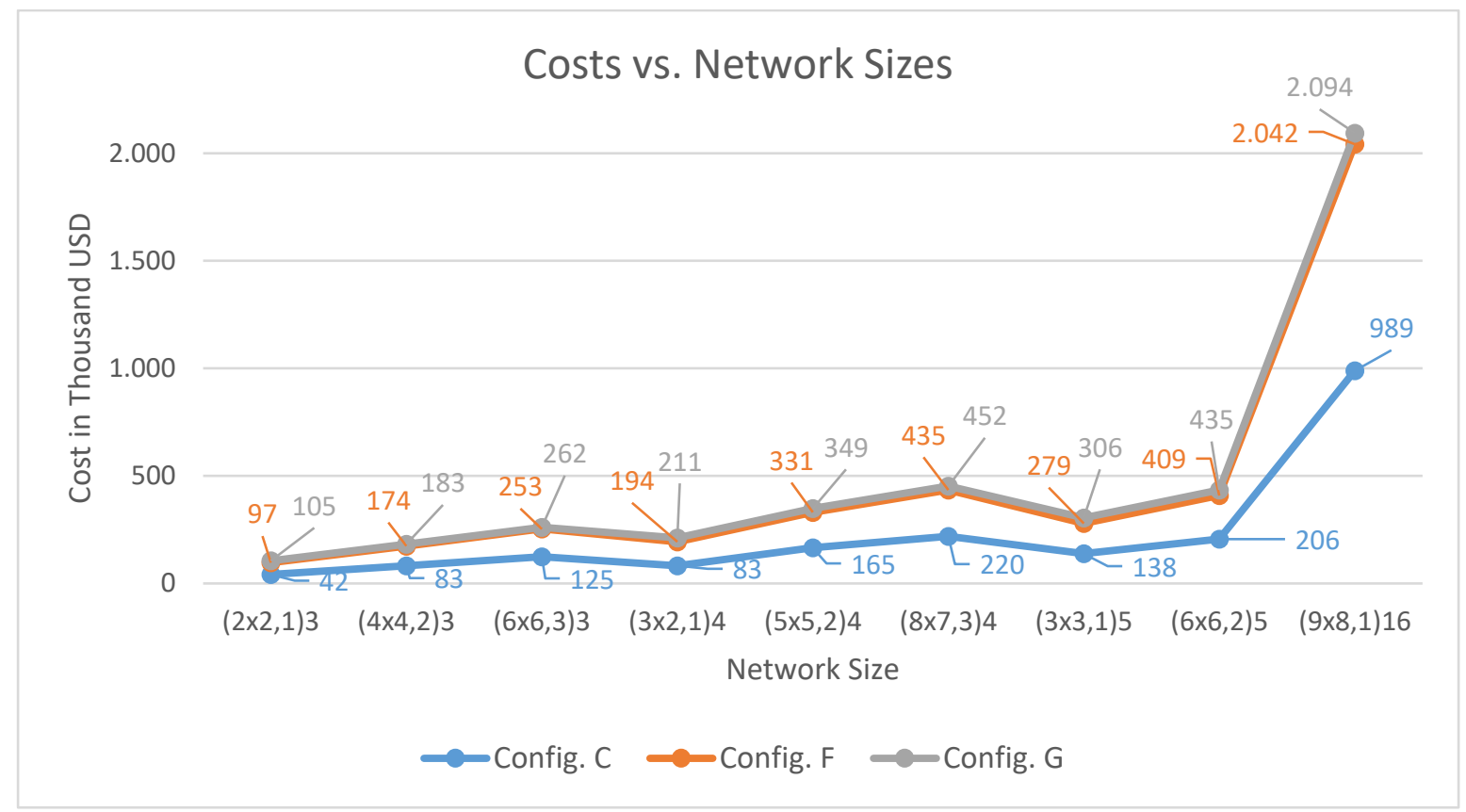

Figure 3. Costs, expressed in thousands of US dollars, of selected configurations from Table 1 vs. network sizes.

\section{IA Testbed Platforms and Experiments}

The number of solutions devoted to experimental research in wireless communications is growing every year, particularly with the increased interest on wireless sensor networks (WSNs) and internet of things (IoT) from the research community. Publicly accessible testbed facilities are great opportunities for IA researchers to experimentally evaluate their algorithms. However, among the large-scale testbed facilities which offer public access for researchers to execute automated and manageable experiments, only few of them are suitable for physical-layer experimentations. A good example is CorteXlab at the University of Lyon, which contains a mixture of low-power, general-purpose and real-time highperformance nodes. Other small-scale publicly available facilities are CREW and CORE+ project consortiums.

There are different platforms available for the implementation of IA testbed. National Instruments and Ettus USRPs together with the open-source universal hardware driver and GNU Radio are among the preferred choices for low-budget cases. Open-source implementations of communication standards like openLTE for the case of LTE and the enormous user community makes this solution very attractive. At an increased cost, National Instruments USRPs can be preferred since they support LabView, a powerful software which is again a proprietary product of National Instruments. Other examples of low-cost solutions available in the market are RTL-SDR, HackRF, and Nuand BladeRF. Unfortunately, their simple designs and limited capabilities make complex IA implementations on these platforms infeasible.

When the budget is not a severe constraint, high-end PXI-based products are much better solutions. There are several PXI hardware vendors, such as Keysight and National Instruments, where the main advantage of National Instruments solutions lies on the software side, specially their integration with LabView and with other powerful software tools like MATLAB from Mathworks.

Many manufacturers offer hardware equipment besides the integrated solutions, allowing for developing a part-by-part testbed. 4DSP, Nutaq, and Innovative Integration are just some examples. Some of the manufacturers offer modules for Xilinx-based boards, and typically they do not provide full solutions or open-source drivers. At a lower level, Texas Instruments, Maxim Integrated or Analog Devices are 
among the manufacturers continuously offering better components for SDR solutions. For example, Analog Devices has introduced a transceiver (AD-FMCOMMS5-EBZ) with up to 8 antennas and 56 $\mathrm{MHz}$ bandwidth on a single board. At a much higher level, Wireless Open-Access Research Platform (WARP) is an example of a full bundle of solutions built from the ground up with the aim of prototyping advanced wireless networks. Several alternatives similar to WARP can be found within the wireless research community, for example the SDR4All project from Supélec.

Frequently, research institutions opt for developing their testbeds based on commercial off-the-shelf hardware, sometimes combined with custom-designed parts. Examples of such testbeds are those developed by the Vodafone Chair for Mobile Communications Systems at the Techsnische Universität Dresden, KTH Testbed, OpenAirInterface at Eurecom, the so-called Vienna MIMO Testbed developed at the Institute of Telecommunications at the Technische Universität Wien, and the one developed at the Heinrich Hertz Institute at the Fraunhofer Institute for Telecommunications.

Once the hardware required to experimentally evaluate IA techniques is ready, a challenge that arises is the generation of a representative and sufficiently large amount of channel realizations guaranteeing repetitive and reproducible results. Fortunately, many clever and inspiring approaches are found in the literature, like for example by considering antenna switching instead of reconfigurable antennas [11], utilizing two different reconfigurable antenna architectures that use different patterns, or simply sliding the receive antenna.

The aforementioned experiments rely on physical techniques, i.e., different antenna architectures, to supply independent channel realizations. However, independent channel realizations can also be created by simply conjugating the complex-valued input and output signals, thus IA can be achieved via the conjugate operation in the static single-input single-output (SISO) X networks without any DoF loss, and in interference networks with some DoF loss.

\section{FUTURE REQUIREMENTS}

In this section we provide an overview on the future requirements to transform IA to an attractive solution to be considered for the next generations of wireless communication systems.

\section{Scalability and Measurements}

As mentioned before, existing experimental setups cover simplified scenarios with a reduced number of nodes and antennas per node. However, real-world wireless networks usually include a large number of users and base stations equipped with several antennas each. Even though the implementation of sophisticated nodes is much more expensive, this aspect is of utmost importance regarding the evaluation of IA in realistic scenarios. Experimental evaluation of wireless communication systems in general requires certain measurement concepts and techniques such as the treatment of uncertainties in the results [12]. As the network size scales, the application of this discipline becomes more complex and also more vital, and hence such measurement concepts and techniques need to be adapted to the particular case of IA as well.

\section{Different Network Topologies}

IA testbed implementations summarized in the previous sections can be gathered under two groups: IA with channel state information at the transmitters (CSIT) in interference networks and IA with no CSIT, e.g. BIA in broadcast networks.

Another promising direction is the implementation of IA in relay networks, either with or without CSIT. For the former, innovative techniques are emerging, such as aligned network coding and aligned interference neutralization. For the later, BIA in relay networks is particularly appealing since IA drawbacks in broadcast or $\mathrm{X}$ networks are eliminated via the relay nodes. For conventional SISO 
interference channels (SISO-ICs) without relays, time-varying channels with long symbol extensions are required to obtain the optimal DoF. This requires an overwhelming amount of channel feedback overhead to each transmitter. However, in the relay-aided SISO-IC, only two time slots are required and hence the feedback overhead is greatly reduced. Moreover, the relays are located in between the transmitters and the receivers, thus have a more accurate CSI feedback from the receivers compared to the CSI feedback to the transmitters, which are located farther from the receivers. Hence, the relay-aided BIA schemes are appealing from a practical perspective. Finally, as mentioned above, extending the BIA schemes to interference broadcast relay channels is also important to serve more users with a less number of base stations deployed in a cellular network.

Nevertheless, regarding the next-generation communications, more complex and advanced network topologies, such as heterogeneous networks (HetNets), should also be considered. Some of the HetNet scenarios seem to be addressable in terms of IA testbed implementation. For instance, a reverse TDD (R-TDD) scenario could be deployed using relatively small and simple nodes. A 2-cell R-TDD setting with these characteristics would be challenging, especially in the CSI feedback aspect, but a combination of IA with non-coherent approaches (e.g., Grassmannian signaling) could also be considered in order to overcome this issue. Altogether, a successful implementation of IA techniques for these kinds of topologies would be a significant step forward in terms of the feasibility of alignment-based transmissions for the next-generation wireless communications.

\section{The Multi-Stream Milestone}

Multi-stream transmissions are essential in modern wireless communication systems, for example for the transmission of rich media. However, they have to consider more aspects compared to their singlestream counterpart. Two good examples are the increased number of quality of service (QoS) parameters, such as multi-bitrate streaming, and self-interference due to multiple information streams for each user. Unfortunately, very few IA experimental evaluations have been carried out considering multi-stream scenarios (e.g. [13]). One reason is that the implementation cost of multi-stream transmission is folded as illustrated in Fig. 3. Moreover, to properly address the experimental evaluation of the throughput of a multi-stream (and also a single-stream) scenario, a minimal MAC layer implementation is necessary. A different approach would be to properly evaluate the performance of the physical layer and later on plug in the results in a network emulator which can transmit real-world data.

\section{Outdoors, High Mobility, and Reverberation Chambers}

Few IA testbed implementations considered outdoor and/or mobile environments, whereas the majority only addressed static indoor scenarios. To the authors' knowledge, there is only one IA testbed implementation that is close to a prototype stage since IA beams are physically transmitted over-the-air and the complete system is realized in real-time [5]. Nowadays, testbed equipment can be powered by small batteries and controlled using a laptop (e.g. B-family USRPs), thus making possible to assess IA in high-mobility scenarios in near future (see [14]).

Reverberation chambers provide repeatable emulations of reasonably realistic conditions at relatively affordable costs. Similar to the success story of the WARP platform, which was initially developed at Rice University that later on gave way to the spin-off company Mango Communications, the OTA reverberation chamber was initiated at Chalmers University of Technology for research purposes and then the technology was transferred to the spin-off company Bluetest $\mathrm{AB}$. 


\section{Software Requirements}

Until now, the discussions are typically dominated by hardware specifications. However, a common repository to share and improve open-source IA software by the developers is perhaps another immediate need. Such an approach can provide a huge momentum for in-depth research and for the expansion of IA applications.

\section{REFLECTIONS ON FUTURE DIRECTIONS}

As stated above, IA offers two main routes in theory: IA with CSIT, and IA with no CSIT or, shortly, BIA. While both IA schemes still face the perfect synchronization and imperfect hardware hurdles, BIA, especially in relay networks, is promising since the drawbacks -CSIT and unlimited network resourcesare eliminated via relay nodes. However, the high SNR requirement -another major IA drawbackremains in both IA schemes. Many complex schemes are proposed to improve the mid-SNR performance of IA at the cost of impairing its simplicity. Relay nodes can be easily utilized to improve the mid-SNR performance of IA as well. In relay networks, BIA is presently known to be more compatible than IA with CSIT.

\section{Promising Directions}

Among the options of the relay-aided SISO-IC scheme [15] as discussed further in the next section, the option of multiple single-antenna relays -each relay has a single-antenna- is less favorable since it has two requirements: joint beamforming between transmitters and relays, and time-varying channels. On the other hand, the option of a single relay with multiple antennas is more favorable since it does not impose those requirements. However, this option can only be favorable for mid-sized networks, e.g., in a 21-user relay-aided SISO-IC, a single relay with 20 antennas can achieve IA. However, for a larger network, e.g., in a 100-user relay-aided SISO-IC, IA can be achieved either via a single relay with 99 antennas or via 25 relays with 20 antennas each. Relay-aided MIMO-ICs can also be preferred for midsized networks since multiple antennas at the transmitters and receivers increase the DoF, but also increase the number of antennas at the relay. When each of the transmitters and receivers has two antennas, a single relay with 20 antennas can achieve IA in an 11-user MIMO-IC. However, 30 antennas are needed at the relay when each of the end nodes has three antennas.

IA techniques are currently difficult to implement in cellular networks, at least not in medium term. Hence massive MIMO, HetNets, and even IoT in cellular networks seem to be unpromising as an immediate industrial pursue for IA. In massive MIMO networks, the massive amount of nodes and antennas imply massive CSI needs. BIA can be implemented in such networks where massive MIMO nodes are the relay nodes in the next generation of cellular systems right after the launch of the massive MIMO technology. Other possible technology options to choose from can be IoT in a future Bluetooth release or in Wi-Fi HaLow networks, green networks, energy harvesting, Li-Fi, body area networks (BAN), and sensor networks. As a particular application, relay-aided BIA can be fine-tuned to be utilized at homes or in commercial airplanes (IoT), on persons (BAN), in solar panels or in smart farms (sensor networks), in airplanes, trains, ships or cars (sensor networks), and in server rooms (green networks) to connect and monitor communicating wireless devices. After choosing the most compatible application for relay-aided BIA, theoretical and experimental-based research must progress in parallel at the same pace so that frequent feedbacks between them expedite the marketization process of IA.

\section{Relay-Aided IA Schemes}

The relay-aided IA scheme utilizes the conventional IA concept along with simply counting the number of variables and equations in the system. The concept is illustrated for the 3-user SISO interference relay channel in Fig. 4. Since it is a two time-slot scheme, each receiver has a 2-dimensional space. Hence $K$ - 
1 interfering signals need to be aligned in a 1-dimensional space. Without loss of generality, this can be achieved as follows. For receiver 1, the normalized interference (NI) from transmitter 2 can be equated to the normalized interference from each of the other transmitters: NI from $\mathrm{Tx}_{2}=\mathrm{NI}$ from $\mathrm{Tx}_{j}, \forall j=$ $\{1,2, \ldots, K\} \backslash\{1,2\}$ at $\mathrm{Rx}_{1}$. For each of the other receivers, the normalized interference from transmitter 1 can be equated to the normalized interference from each of the other transmitters: NI from $\mathrm{Tx}_{1}=$ NI from $\mathrm{Tx}_{j}, \forall j=\{1,2, \ldots, K\} \backslash\{1, i\}$ at $\mathrm{Rx}_{i}, \forall i=\{1,2, \ldots, K\} \backslash\{1\}$. This is illustrated in Fig. 4 via the colored channels for the communication to receiver 1 through relay 1 only. The IA equations of the system can be reformulated in a matrix structure $\mathbf{H} \boldsymbol{\alpha}=\mathbf{z}$ as defined in Fig. 4. The core idea of the proposed scheme is that the $\mathbf{z}$ vector cannot be zero when the number of constraints (i.e., number of rows in $\mathbf{H}$ ) is equal to the number of variables (i.e., number of columns in $\mathbf{H}$ ). Otherwise, since $\mathbf{H}$ is invertible, the scaling factors of relays are all zero, $\boldsymbol{\alpha}=\mathbf{0}$. Therefore, the transmitters during the second time slot cannot be silent (i.e., $a_{i}^{\prime}$ s cannot be zero) as well as the channels must be time varying (i.e., $a_{i}^{\prime}$ cannot be equal to $\left.a_{i}\right)$. Since there are $K(K-2)$ IA equations in total, there need $K(K-2)$ relays in the system so that there are $K(K-2)$ variables (i.e., scaling factors) as also proposed in [15]. However, as illustrated in Fig. 4, with the addition of a relay, $\mathbf{H}$ is not square, hence $\mathbf{z}$ vector can be zero, meaning that joint beamforming (i.e., transmitters also transmit during the second time slot) and time-varying channels are not needed.

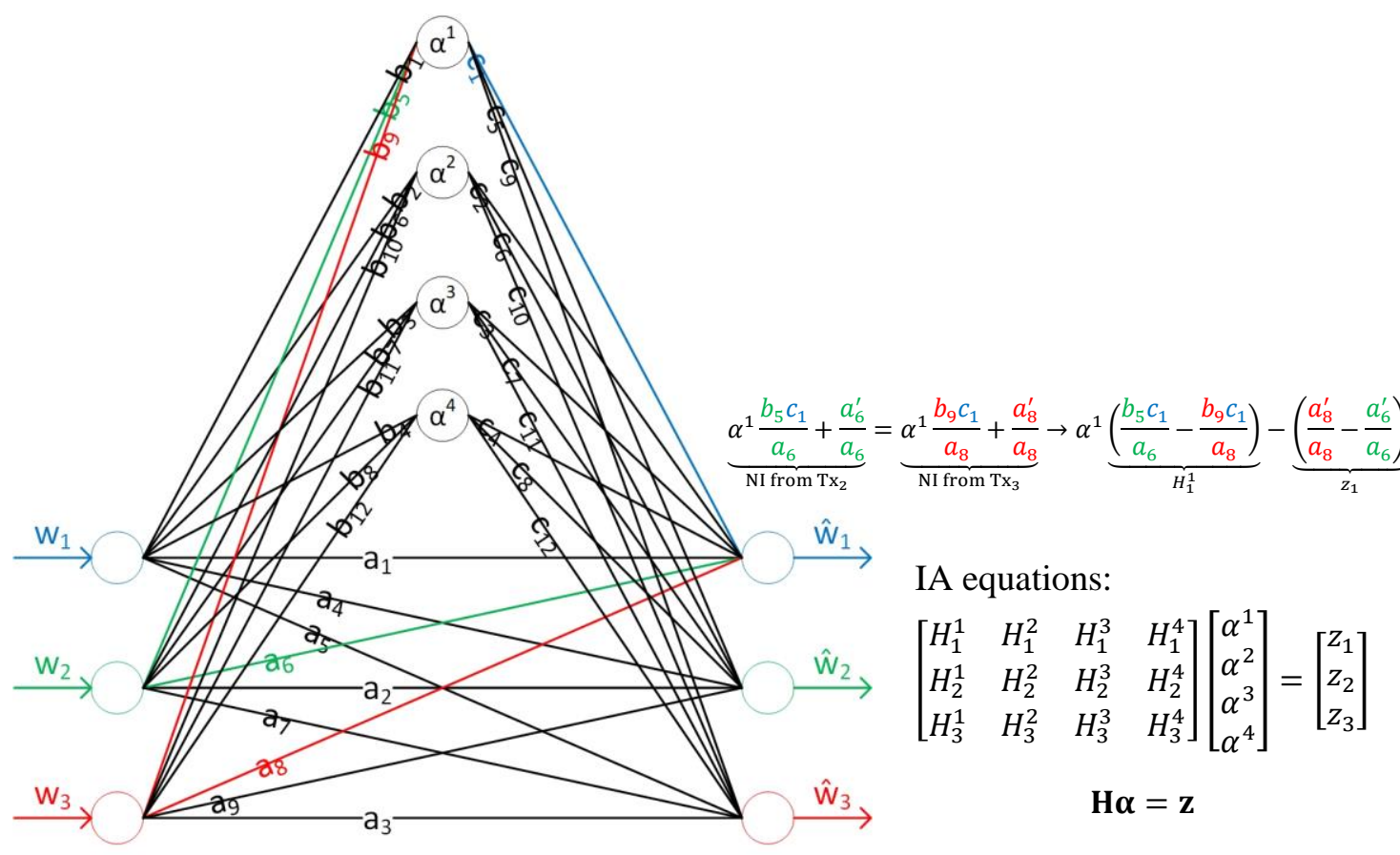

Figure 4. 3-user relay-aided SISO-IC. $\alpha^{q}$ is the scaling factor of the $q^{\text {th }}$ relay. $a_{i}$ and $a_{i}^{\prime}$ are the direct channels in the first and second time slots, respectively. $b_{i}$ and $c_{i}$ are the channels between the transmitters and relays, and the relay and receivers, respectively. The indirect channels are subindexed so that the desired and interference signals are carried over the effective channels $b_{i} c_{i}$ and $b_{i} c_{j}, j \neq i$, respectively. $H_{i}^{q}$ is the difference of the normalized interferences received via relays, $\forall i=$ $\{1,2, \ldots, K\}$ and $\forall q=\{1,2, \ldots, Q\}$, where $Q$ is the total number of relays in the system. $z_{i}$ is the difference of the normalized interferences received via direct channels in the second time slot. 


\section{CONCLUSION}

In this article, a survey on IA testbed implementations has been presented. Highlights, challenges, and future directions of IA experimentations are provided from a broad perspective. Testbed experiments on the feasibility and performance of IA schemes have demonstrated significant gains compared to more conventional schemes. However, IA experimentation is still in its infancy, leading to a big gap between theoretical and experimental progresses. Moreover, IA testbed platforms notably lack many components compared to other worldwide deployed testbeds that have sophisticated configurations and features. More collaborations with both computer scientists and engineers as well as with other specialists in electronics, including microelectronics, can expedite the delivery date of IA to real-life.

\section{ACKNOWLEDGEMENT}

Jacobo Fanjul's research has been supported by the Ministerio de Economía y Competitividad (MINECO) of Spain, under grants TEC2013-47141-C4-R (RACHEL project) and FPI grant BES-2014069786. José A. García-Naya's research has been funded by the Xunta de Galicia (ED431C 2016-045, ED341D R2016/012, ED431G/01), the Agencia Estatal de Investigación of Spain (TEC2013-47141C4-1-R, TEC2015-69648-REDC, TEC2016-75067-C4-1-R) and ERDF funds of the EU (AEI/FEDER, UE). Hamed Farhadi's research has been funded by the Swedish Research Council (VR), under grant 2015-00500.

\section{REFERENCES}

[1] H. Farhadi, M. N. Khormuji, and M. Skoglund "Pilot-assisted ergodic interference alignment for wireless networks," in Proc. of the 2014 IEEE International Conference on Acoustics, Speech and Signal Processing (ICASSP), pp.6186-6190, 4-9 May 2014.

[2] S. Gollakota, S. D. Perli, and D. Katabi, "Interference alignment and cancellation," in Proc. of ACM Special Interest Group on Data Comm (SIGCOMM), Aug. 17-21, 2009.

[3] C. Lameiro, O. González, J. A. García-Naya, I. Santamaría, and L. Castedo, "Experimental evaluation of interference alignment for broadband WLAN systems", in EURASIP Journal on Wireless Communications and Networking, vol. 2015, issue 180, June 2015.

[4] J. Fanjul, C. Lameiro, I. Santamaria, J.A. Garcia-Naya, and L. Castedo, “An experimental evaluation of broadband spatial IA for uncoordinated MIMO-OFDM systems," in Proc. of the IEEE International Conference on Digital Signal Processing (DSP), pp. 570-574, 21-24 July 2015.

[5] S. Lee, A. Gerstlauer, and R. W. Heath, "Distributed real-time implementation of interference alignment with analog feedback," in IEEE Transactions on Vehicular Technology, vol.64, no.8, pp.3513-3525, Aug. 2015.

[6] O. El Ayach, A. Lozano, and R. W. Heath, "On the overhead of interference alignment: training, feedback, and cooperation," in IEEE Transactions on Wireless Communications, vol. 11, no. 11, pp. 4192-4203, November 2012.

[7] N. N. Moghadam, H. Farhadi, and P. Zetterberg, "Optimal power allocation for pilot-assisted interference alignment in MIMO interference networks: Test-bed results," in Proc. of the 2015 IEEE International Conference on Digital Signal Processing (DSP), vol., no., pp.585-589, 21-24 July 2015.

[8] M. El-Absi, S. Galih, M. Hoffmann, M. El-Hadidy, and T. Kaiser, "Antenna selection for reliable MIMO-OFDM interference alignment systems: Measurement based evaluation," IEEE Trans. Veh. Technol., vol. 65, no. 5, pp. 2965-2977, May. 2015. 
[9] P. Zetterberg and N. Moghadam, "An experimental investigation of SIMO, MIMO, interferencealignment (IA) and coordinated multi-point (CoMP)," in Proc. of the International Conference on Systems, Signals and Image Processing (IWSSIP), pp. 211-216, 11 - 13 April 2012.

[10] H. Farhadi, C. Wang, and M. Skoglund, "Distributed transceiver design and power control for wireless MIMO interference networks," in IEEE Transactions on Wireless Communications, vol. 14, no. 3, pp. 1199-1212, Mar. 2015.

[11] K. Miller, A. Sanne, K. Srinivasan, and S. Vishwanath, "Enabling real-time interference alignment: Promises and challenges," Mobile Ad Hoc Networking and Computing (MobiHoc), pp. 55 - 64, 11 - 14 June 2012.

[12] S. Caban, J. A. Garcia-Naya, and M. Rupp, "Measuring the physical layer performance of wireless communication systems: Part 33 in a series of tutorials on instrumentation and measurement," in IEEE Instrumentation and Measurement Magazine, vol. 14, no. 5, pp. 8-17, Oct. 2011.

[13] G. Artner, M. Mayer, M. Guillaud, and M. Rupp, "Measuring the impact of outdated channel state information in interference alignment techniques," Sensor Array and Multichannel Signal Process. Workshop Proceedings, pp. 353 - 356, 22 - 25 June 2014.

[14] J. Rodríguez-Piñeiro, M. Lerch, J. A. García-Naya, S. Caban, M. Rupp, and L. Castedo, "Emulating extreme velocities of mobile LTE receivers in the downlink," in EURASIP Journal on Wireless Communications and Networking, vol. 2015, issue 106, April 2015.

[15] Y. Tian and A. Yener, "Guiding blind transmitters: Degrees of freedom optimal interference alignment using relays,” IEEE Trans. Inf. Theory, vol. 59, no. 8, pp. 4819-4832, Aug. 2013.

\section{BIOGRAPHIES}

CENK M. Yetis [S’00, M'10] (cenkmyetis@ieee.org) received the B.Sc.'01 from Isik University, M.Sc.'04 and Ph.D.'10 both from Istanbul Technical University in Turkey. From 2003 to 2007, he worked as an engineer in a wireless service provider in Turkey. From 2007 to 2010, he was a visiting researcher in US. From 2010 to 2016, he held academic positions in Universities in Hong Kong, Singapore, and Turkey. In 2016, he joined Academia Sinica in Taiwan. His research interests include signal processing, information, communication and optimization theories for wireless communications.

JACOBO FANJUL [S'13] (fanjulj@unican.es) received his Telecommunication Engineering (M.Sc.) degree from the University of Cantabria, Santander, Spain, in 2014. In 2013, he joined the Department of Communications Engineering, University of Cantabria, where he is currently pursuing his Ph.D. in Electrical Engineering. During 2016, he was a visiting researcher at the Department of Electrical Engineering and Computer Science (EECS), University of California, Irvine. His current research interests include signal processing algorithms for interference alignment, heterogeneous networks (HetNets), MIMO testbeds and interference management for noncoherent wireless communication.

JosÉ A. GARCíA-NAYA [S'07, M'11] (jagarcia@udc.es) studied Computer Engineering at the University of A Coruña (UDC), Spain, where he obtained his M.Sc. degree in 2005, and his Ph.D. degree in 2010. Since 2005 he is with the Group of Electronics Technology and Communications (GTEC) at the UDC, where he is currently Associate Professor. His research interests are in the field of wireless communication systems, with special emphasis on their experimental evaluation.

NimA N. MoghaDAM [S'12] (nimanm@kth.se) received the B.S. degree in electrical engineering from Shahid Beheshti University, Tehran, Iran in 2008 and the M.S. degree in wireless systems from KTH 
Royal Institute of Technology, Stockholm, Sweden in 2010, where he is currently pursuing the Ph.D. degree. His research interests lie in the area of wireless communication with emphasis on multiantenna cellular communications, radio resource allocation and software-defined radio.

HAMED FARHADI [S'06-M'15] (farhadi@seas.harvard.edu) received the $\mathrm{PhD}$ degree in Telecommunication from KTH Royal Institute of Technology, Stockholm, Sweden, in 2014. He has been a Postdoctoral Research Fellow at John A. Paulson School of Engineering and Applied Sciences, Harvard University, MA, USA since 2016, and a Researcher at the Department of Signals and Systems, Chalmers University of Technology, Gothenburg, Sweden since 2015. Dr. Farhadi is an Associate Editor of the Springer International Journal of Wireless Information Networks. 